\title{
The Research of Corner Extractors in Local Feature Points Matching Algorithm
}

\author{
J.B. Zhi, F.C. You \\ College of Information Engineering \\ Beijing Institute of Graphic Communication \\ Beijing, China
}

\begin{abstract}
Local feature extraction is the key point of local feature points matching algorithms. Most of the feature points extractors can be categorized into corner extractors, blob extractors or region extractors. Corner extractors become a research hotspot in recent years due to its simple structure and accuracy. At first, the flow of local feature points matching algorithm is introduced, and the properties and the categories of the local feature points are also introduced. Secondly, this paper sets focus on several important corner extractors and new progress of the local feature points matching algorithms based on the Features from Accelerated Segment Test(FAST) corner extractor. Finally, some research challenges and directions are discussed.
\end{abstract}

Keywords- local feature points; local feature points matching algorithm; feature extraction; corner feature

\section{INTRODUCTION}

Local feature points matching algorithm is widely used in many computer vision applications in recent years. Object recognition, image registration, camera calibration are just a few. The first local feature points matching algorithm was introduced by Moravec[1] in 1977. After nearly 40 years of development, many excellent algorithms have been proposed. The local feature point should not be simply interpreted as an isolated point, it can actually be a corner, a blob or even a region based on the local structure of an image. Therefore, most of the local feature points matching algorithms can be categorized into corner-based, blob-based and region-based matching algorithms. In this paper, we briefly introduce the local feature points matching algorithm, and then focus on some important corner extractors (Harris, SUSAN, FAST) in the process of feature points extraction and briefly introduce some new progresses of the local feature points matching algorithms based on the FAST corner extractor in recent years.

\section{LOCAL FEATURE POINTS MATCHING ALGORITHM}

Local feature points matching algorithm can be divided into three stages: feature points extraction, feature points description and descriptors matching. Firstly, a certain amount of local feature points need to be extracted respectively from the original image and the distorted image. Secondly, we describe each local feature point to generate a feature vector. Finally, the two feature vectors are matched so that we can get the spatial location relation between the two images. Figure 1 shows the flow chart of the local feature points matching algorithm.

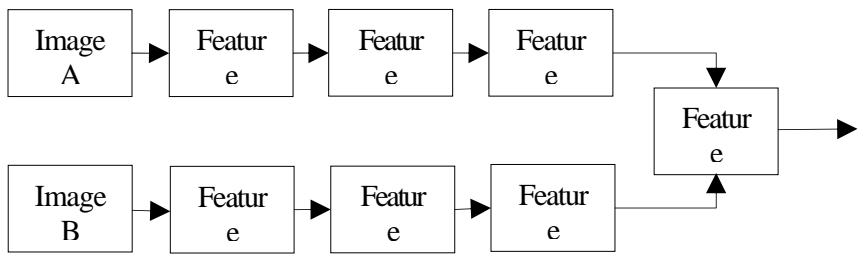

FIGURE I . FLOW CHART OF THE LOCAL FEATURE POINTS MATCHING ALGORITHM.

\section{Properties AND CATEgORIES OF LOCAL FEATURE POINTS}

\section{A. Properties of the Local Feature Points}

We would like to such local feature points to correspond to semantically meaningful object parts. But it is unfeasible, because extractors usually select feature points directly based on the underlying intensity patterns. However, good local feature points should have the following properties[2]: (1) repeatability: a high percentage of the feature points extracted on the same scene should be found in images which may be taken under different viewing conditions; (2) robustness: the selected local feature points should be less sensitive to some deformations such as noise, blur and compression artifacts; (3)efficiency: the process of local feature points extraction should be as quick as possible; (4) invariance: the same local feature points can still be extracted even when some large deformations are to be expected; (5) accuracy: local feature points extracted should be accurately localized both in image space and in scale space.

\section{B. Categories of Local Feature Points}

Local feature points extraction is the precondition of local feature points description. According to the local structure of the image, feature points can be divided into three categories: (1) corner: corner is probably the simplest kind of local feature points and is often seen as the corner of real world objects. (2) blob: it is a narrow area in the image which is often extracted as local maxima of some certain filters. (3) region: it refers to flat area in the image, this means that one should extract not just a point but a local neighborhood of a pixel. 


\section{CORNER EXTRACTORS}

Corners are often found at various types of junctions such as $\mathrm{L}, \mathrm{T}$ and $\mathrm{X}$. It is convenient to extract corners in many practical applications. Therefore, corner-based extractors become a research hotspot in recent years due to its simple structure and accuracy. Except from Moravec, many other extractors, such as Harris, SUSAN and FAST have been proposed.

\section{A. Harris Extractor}

Harris extractor was proposed by Harris and Stephens[3] in 1988. It computes a corner response based on autocorrelation matrix which is often used to describe the gradient distribution in a local neighborhood of a point. If a small window $W(x, y)$ is translated $(\Delta x, \Delta y)$ across the image $I(x, y)$, the auto-correlation $s$ can be written as follows:

$$
s(x, y, \Delta x, \Delta y)=\sum_{(u, v) \in W(x, y)} w(u, v)(I(u, v)-I(u+\Delta x, v+\Delta y))^{2}
$$

Where, point $(x, y)$ is the center of the window $W(x, y)$, $W(u, v)$ is Gaussian weight. Taylor expansion is applied to simplify the computation, and eqn.(1) can then be written as follows:

$$
s(x, y ; \Delta x, \Delta y)=[\Delta x, \Delta y] M(x, y)\left[\begin{array}{l}
\Delta x \\
\Delta y
\end{array}\right]
$$

with

$$
M(x, y)=\sum_{w}\left[\begin{array}{cc}
I x^{2} & I x I y \\
I x I y & I y^{2}
\end{array}\right]
$$

In order to improve the efficiency further, Harris gives a corner response $R$ which does not need to compute eigenvalues of the matrix $\mathrm{M}$ :

$$
R=\operatorname{det} M-\alpha(\operatorname{trace} M)^{2}
$$

where $\operatorname{det} M$ and $\operatorname{trace} M$ are respectively the determinant and the trace of the matrix $M, \alpha$ is often experimentally $0.04 \sim 0.06$. Corner can then be found when $R$ is large enough.

Due to the image differential operation, Harris extractor is robust to illumination and contrast variation. Harris corner is also translation and rotation invariant. Moreover, subpixel precision can be achieved through quadratic approximation of the function. But the shortcomings of the algorithm are also very obvious. The computation is still expensive though eigenvalues are avoided to be computed. What is worse, Harris is sensitive to scale variation[4].

\section{B. Susan Extractor}

To solve the problem of slow speed of Harris extractor, SUSAN (Smallest Univalue Segment Assimilating Nucleus) extractor was proposed by Smith and Brady [5] in 1997. Unlike the Harris, SUSAN extractor uses the theory of morphology. Utilizing a circular template and the center of this circle, the pixel value of the center is compared with the other pixel values within the circle, and the pixels that are similar to the center one are counted. If the number is smaller than the defined threshold, the center pixel is the corner.

When the circle template moves on the image, the result $n$ that the center pixel value is compared with the other pixel values within the circle is defined by simple binary value:

$$
n\left(p, p_{0}\right)= \begin{cases}1, & \left|I(p)-I\left(p_{0}\right)\right| \leq t \\ 0, & \left|I(p)-I\left(p_{0}\right)\right|>t\end{cases}
$$

In eqn.(5), $p_{0}$ and $p$ are the center point of the template and the other points of the template in the image, respectively. In addition, $I(\cdot)$ is the pixel gray-value of the corresponding point, and $t$ is the defined threshold. After all the pixels of the circle template are calculated, the statistic result can be written as follows:

$$
S\left(p_{0}\right)=\sum_{p} n\left(p, p_{0}\right)
$$

$S\left(p_{0}\right)$ is the area of the nucleus similarity region of the circle template in the image, and $S\left(p_{0}\right)$ reaches to the local minimum when $p_{0}$ is a corner.

The morphology idea and the statistics characteristic make SUSAN insensitive to the local noise, thus it has strong immunity from interference. By avoiding the image differential operation, the computation complexity of SUSAN extractor is lower than that of the Harris extractor, thus the speed of SUSAN extractor is faster. However, there is weakness of SUSAN: because some detected corners are the edge structures rather than real corners, it leads to the limits in some application field.

\section{Fast Extractor}

FAST(Features from Accelerated Segment Test) extractor was proposed by Rosten et al[6] in 2005, and was improved in subsequent papers[7,8]. It uses a machine learning method to further improve the speed of SUSAN extractor. As can been seen in Figure 2, on the fixed circular template, FAST just only computes the difference value between the sixteen pixels on a circle and the center pixel which do not need to statistic all the pixels within the circular template. Compared with SUSAN extractor, FAST reduces the computational complexity greatly, and in order to speed up the extractor further, only pixels where $1,5,9$, 13 need to be tested to select candidate corners.

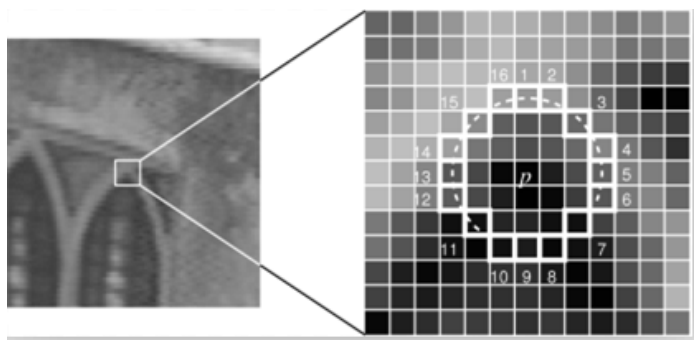

FIGURE II. FAST CORNER EXTRACTOR FROM ROSTEN

For each location on the circle $x \in\{1,2,3 \ldots, 16\}$, it can get a state type by the rule which can be written as follows: 


$$
S_{x}=\left\{\begin{array}{lllll}
d, & & I_{x} & \leq I_{p}-t & \text { (darker) } \\
s, & I_{p}-t< & I_{x} & <I_{p}+t & \text { (similar) } \\
b, & I_{p}+t \leq & I_{x} & & \text { (brighter) }
\end{array}\right.
$$

where, $t$ is the threshold. $I_{p}$ is the gray value of the center pixel. $I_{x}$ represents the gray value of pixels on the circle. When the state $d$ or $b$ on the circle appears continuously to a certain number $\mathrm{n}$, the center pixel is then defined as a corner.

FAST extractor can be divided into three main steps:

1) Perform test on the fixed size circle template to remove non-candidate corners.

2) Screen the candidate corners further using ID3 decision tree.

3) Perform non-maximal suppression for the rest of the candidate corners to select the most stable corners.

Rosten proved by experiments that FAST extractor is more efficient than the Harris extractor and SUSAN extractor. It also has high level repeatability. However, the disadvantages of FAST extractor are also obvious: FAST extractor achieves high level efficiency by analyzing only a few pixels that make it less robust to noise; FAST extractor is also weak in the robustness to scale change.

\section{New Progress of Fast-Based Local Feature Points Matching Algorithm}

In recent years, FAST becomes one of the most popular feature points extractor because of its efficiency. Many excellent local feature points matching algorithms have been proposed based on modified FAST extractor, which makes the real-time computer vision application to achieve substantial development.

Mair proposed The AGAST(Adaptive and Generic Accelerated Segment Test)[9] in 2010, which is faster and better than FAST. AGAST modifies the low-level decision trees of the FAST. ID3 is used to build the decision tree in FAST, which is a greedy algorithm and, therefore, the result can be quite suboptimal. While AGAST uses the binary tree instead of the ID3, which will be more efficient than FAST. By combining two trees, Adaptive and Generic Accelerated Segment Test(AGAST) does not have to be trained while preserving the same corner response and repeatability as the FAST extractor. As can be seen in the experiments of Mair, compared with FAST9-16, FAST7-12 and FAST5-8, AGAST respectively achieves speed-up of about $13 \%$, 30\% and $50 \%$.

AGAST is faster than the original FAST extractor, but it can not solve the problem that FAST is sensitive to scale variation. However, S. Leutenegger[10] gave the solution in BRISK(Binary Robust Invariant Scalable Keypoints) in 2011. In BRISK, corners are detected in octave layers of the image pyramid as well as in layers in-between. Both the location and the scale of each corner are further obtained in the continuous domain via quadratic function fitting. It shows that in the experiments of S. Leutenegger, the BRISK exhibits equivalent repeatability as SIFT and SURF, but more efficient than both of them.

\section{E. Discussion}

As members of corner extractors, Harris, SUSAN and FAST are all have the advantages of high accuracy and high repeatability. Harris corner is more stable than SUSAN corner and FAST corner. However, because of the image differential operation, Harris extractor is less efficient than the other two extractors. Table 1 shows some properties of Harris, SUSAN and FAST.

TABLE I. PROPERTIES OF HARRIS, SUSAN AND FAST.

\begin{tabular}{|l|l|l|l|l|}
\hline Extractor & $\begin{array}{l}\text { Efficienc } \\
\mathrm{y}\end{array}$ & Accuracy & $\begin{array}{l}\text { Robustne } \\
\text { ss }\end{array}$ & $\begin{array}{l}\text { Repeatabi } \\
\text { lity }\end{array}$ \\
\hline Harris & + & +++ & +++ & +++ \\
\hline SUSAN & ++ & +++ & ++ & ++ \\
\hline FAST & +++ & ++ & ++ & +++ \\
\hline
\end{tabular}

\section{CONCLUSIONS}

Throughout the development of feature point matching of past decades, the strong robustness and high speed algorithms are the key trend. Particularly, the local feature matching algorithms that are based on corner detection make substantial progress in this field. But speed, accuracy and the robustness to various interferences are still the key point of the study in feature point matching algorithms. It is hard to select only one local feature to process all the images in all situations. It is a great challenge to find the suitable local feature adaptively according to the property of images. In a word, one of the study tendencies of local feature points matching is multi-features fusion.

\section{ACKNOWLEDGEMENTS}

This paper is supported by Scientific Research Key Project of Beijing Municipal Commission of Education (KZ201210015015)and Beijing Natural Science Foundation (4122026).

\section{REFERENCES}

[1] Moravec H. Towards automatic visual obstacle avoidance. Proceedings of International Joint Conference on Artificial Intelligence, New York, USA: ACM Press, pp. 584, 1977.

[2] Tuytelaars T, Mikolajczyk K. Local invariant feature detectors:a survey. Foundations and Trends in Computer Graphics and Vision, 3(3), pp. 177-280, 2007.

[3] Harris C, Stephens M.A combined corner and edge detector Proceedings of Alvey Vision Conference, New York, USA:ACM Press, pp. 147-151, 1988.

[4] Mikolajczyk K, Schmid C. Scale\&affine invariant interest point detectors. International Journal of Computer Vision, 60(1), pp. 63-86, 2004.

[5] Smith S.M, Brady J.M.SUSAN-a new approach to low level image processing. International Journal of Computer Vision, 23(1), pp. 45-78, 1997.

[6] Rosten E, Drummond T. Fusing points and lines for high performance tracking. Computer Vision, 2005. ICCV 2005. Tenth IEEE International Conference on. IEEE, 2, pp. 1508-1515, 2005.

[7] Rosten E, Drummond T. Machine learning for high-speed corner detection. Computer Vision-ECCV 2006. Springer Berlin Heidelberg, pp. 430-443, 2006.

[8] Rosten E, Porter R, Drummond T. Faster and better: A machine learning approach to corner detection. Pattern Analysis and Machine Intelligence, IEEE Transactions on, 32(1), pp. 105-119, 2010. 
[9] Mair E, Hager G D, Burschka D, et al. Adaptive and generic corner detection based on the accelerated segment test. Computer VisionECCV 2010. Springer Berlin Heidelberg, pp. 183-196, 2010.

[10] Leutenegger S, Chli M, Siegwart R Y. BRISK: Binary robust invariant scalable keypoints. Computer Vision (ICCV), 2011 IEEE International Conference on. IEEE, pp. 2548-2555, 2011. 